Preprint typeset in JHEP style - PAPER VERSION

ITFA-2005-50

hep-th/0512125

\title{
Holographic Renormalization of Probe D-Branes in AdS/CFT
}

\author{
Andreas Karch, ${ }^{a}$ Andy O'Bannon ${ }^{a}$ and Kostas Skenderis ${ }^{b}$ \\ ${ }^{a}$ Department of Physics, University of Washington, Seattle, WA 98195-1560 \\ ${ }^{b}$ Institute for Theoretical Physics, University of Amsterdam, Valckenierstraat 65, 1018 XE \\ Amsterdam, The Netherlands \\ E-mail: karch@phys.washington.edu, ahob@u.washington.edu, \\ skenderi@science.uva.nl
}

\begin{abstract}
We perform holographic renormalization for probe branes in $A d S_{5} \times S^{5}$. We show that for four known probe D-branes wrapping an $A d S_{m} \times S^{n}$, the counterterms needed to render the action finite are identical to those for the free, massive scalar in $A d S_{m}$ plus counterterms for the renormalization of the volume of $A d S_{m}$. The four cases we consider are the probe D7, two different probe D5's and a probe D3. In the D7 case there are schemedependent finite counterterms that can be fixed by supersymmetry.
\end{abstract}

KEYwORDs: AdS/CFT, D-branes, holographic renormalization. 


\section{Contents}

$\begin{array}{ll}\text { 1. Introduction } & 1\end{array}$

2. Review: Holographic Renormalization $\quad 2$

3. Generalities for Probe Branes $\quad 6$

3.1 General Solution and Counterterms 6

3.2 Supersymmetric Background Solution 9

4. The D3/D7 System 10

4.1 The System 10

4.2 Counterterms 10

4.3 One-point function 11

5. The D3/D5 System 12

5.1 The System 12

5.2 Mass deformation $\quad 12$

5.3 Vev deformation $\quad 13$

6. The D3/D3 System 14

6.1 The System 14

6.2 Counterterms and One-Point Function $\quad 15$

6.3 Two-point functions 16

\section{Introduction}

Holography equates a $(d+1)$-dimensional theory of gravity with a $d$-dimensional field theory. The AdS/CFT correspondence [1] is an explicit example of this, in which supergravity (SUGRA) formulated on the ten-dimensional bulk spacetime $A d S_{5} \times S^{5}$ is dual to $\mathcal{N}=4$ $S U(N)$ super Yang-Mills (SYM) theory living on the four-dimensional boundary of the $A d S_{5}$ factor. The precise statement of the duality equates the on-shell bulk action with the generating functional of the CFT $[2,3]$. In general, however, both of these quantities are infinite: the bulk action suffers IR divergences while UV divergences appear on the CFT side. The method of holographic renormalization $[4,5,6,7]$ was developed to remove these divergences in a consistent way so that the correspondence equates finite, physical quantities. 
Holographic renormalization involves regulating the on-shell bulk action and then adding covariant counterterms to cancel the divergences that appear as the regulator is removed. This technique is very general, and should certainly apply to the subset of bulk solutions that describe supersymmetric (SUSY) probe brane solutions in AdS/CFT. The AdS/CFT duality is constructed from the near-horizon limit of a stack of $N$ D3-branes with $N \rightarrow \infty$. Introducing a finite number of additional branes orthogonal to the D3's in some directions will produce probe branes embedded in the near-horizon geometry of the D3's, which is $A d S_{5} \times S^{5}$. The simplest known probe branes of this type preserve half the supersymmetry and introduce additional fields in the $\mathcal{N}=4 \mathrm{SYM}$. Their action is the Dirac-Born-Infeld (DBI) action, whose contribution to the bulk action is simply the volume of the brane. Since the backreaction of the probe branes on the background geometry can be neglected this system is probably the simplest application of holographic renormalization in a string theory setting and serves as a nice illustration of the method. One aspect of holographic renormalization for submanifolds, the conformal anomaly, was previously studied in [8]. In this paper, we consider four known probes: a D7-brane [9], two D5-branes [10, 11], and a probe D3-brane [12]. One of the cases in non-supersymmteric [11] while the other three cases are half supersymmetric. The brane embedding in all of these cases is described by a single scalar field that, via the correspondence, will be dual to some operator in the field theory.

Given a renormalized bulk action, renormalized correlators in the dual field theory can be computed straightforwardly. The goal of this paper is to compute renormalized one-point functions, or vacuum expectation values (vev's), for the operators dual to the embedding scalars, using holographic renormalization. Especially for the D3/D7 system the situation is interesting since the vev is not just the subleading term in the asymptotic expansion of the scalar field. Our calculation of the vev clarifies the somewhat ad hoc procedure that has been used in the literature.

This paper is organized as follows. In section 2, we review holographic renormalization, including the methods of adding covariant counterterms to render the bulk action finite and of computing one-point functions from this renormalized action. In section 3 , we show that the counterterms for our DBI actions are identical to the counterterms for a free scalar in $A d S$. In section 4, for the D7, we use these counterterms to compute the one-point function for the embedding scalar's dual operator. In this case, we find that a finite counterterm must be included if the renormalization scheme is to be supersymmetric. We do a similar computation of a one-point function in section 5 for the D5 probe. We also consider a second D5 embedding that is not considered in the general arguments of section 3 but which needs no new counterterms. In Section 6 we compute one- and two-point functions for the D3 probe.

\section{Review: Holographic Renormalization}

We review in this section the holographic renormalization of the volume of an $A d S_{d+1}$ spacetime and of a free scalar in that spacetime. The former has been studied extensively in 
$[4,5,6,13]$, while the latter was explained in detail in [7], see [14] for a review of holographic renormalization.

To begin, write the metric of asymptotically $A d S_{d+1}$ spacetime in the form of Fefferman and Graham [15] in units where the AdS radius is one (which we use throughout):

$$
d s^{2}=G_{\mu \nu} d x^{\mu} d x^{\nu}=\frac{d r^{2}}{r^{2}}+\frac{1}{r^{2}} g_{i j}(x, r) d x^{i} d x^{j},
$$

where $\mathrm{r}$ is the radial coordinate, $\mu, \nu=0, \ldots, d$ and $i, j=0, \ldots, d-1$ and where

$$
g(x, r)=g_{(0)}+r^{2} g_{(2)}+\ldots+r^{d} g_{(d)}+h_{(d)} \log \left(r^{2}\right)+O\left(r^{d+1}\right) .
$$

The logarithmic term only appears for even $d$ and only even powers of $r$ appear up to order $r^{d-1}$. Solving Einstein's equation recursively for the $g_{(i)}$ gives two useful identities ${ }^{1}: \operatorname{Tr} g_{(2)}=$ $\frac{R_{0}}{2(d-1)}$, with $R_{0}$ the Ricci scalar built from $g_{(0)}$, and $\operatorname{Tr} g_{(4)}=\frac{1}{4} \operatorname{Tr}\left(g_{(2)}^{2}\right)[7]$.

For the renormalization of the volume of $A d S_{d+1}$, we restrict our discussion to $d=2,3,4$, which will be relevant for the probe branes we consider. The volume is ${ }^{2}$

$$
V_{A d S}=\int d^{d+1} x \sqrt{G} .
$$

Naively, this volume is infinite since the integration extends all the way to the boundary at $r=0$. Holographic renormalization proceeds in two steps. First, a regulator is introduced by extending the integration only to $r=\epsilon$. Second, counterterms are added to cancel $\epsilon \rightarrow 0$ divergences, yielding a finite, physical answer. These counterterms must be built from data on the $r=\epsilon$ slice to preserve covariance. This will include the induced metric on the $r=\epsilon$ slice, $\gamma_{i j}$, and the Ricci scalar (and tensor) built from $\gamma, R_{\gamma}$. This counterterm procedure is valid for any on-shell bulk action, which will in general be divergent due to integration down to $r=0$, and applies to both the $A d S_{d+1}$ volume and the free scalar action as we will review below. The counterterms for the volume renormalization are

$$
\begin{aligned}
L_{1} & =-\frac{1}{d} \sqrt{\gamma}, \\
L_{2} & =\left\{\begin{array}{cc}
-\frac{1}{2} \frac{1}{d(d-1)(d-2)} \sqrt{\gamma} R_{\gamma} & \text { for } d \neq 2 \\
\frac{1}{4} \log (\epsilon) \sqrt{\gamma} R_{\gamma} & \text { for } d=2
\end{array}\right.
\end{aligned}
$$

and for $d=4$ only, an additional counterterm is needed:

$$
L_{3}=-\log \epsilon \sqrt{\gamma} \frac{1}{32}\left(R_{i j} R^{i j}-\frac{1}{3} R_{\gamma}^{2}\right) .
$$

We will denote subtracted and renormalized quantities as $V_{s u b}=V_{A d S}+\int d^{d} x\left[L_{1}+L_{2}+L_{3}\right]$ and $V_{\text {ren }}=\lim _{\epsilon \rightarrow 0} V_{\text {sub }}$.

\footnotetext{
${ }^{1}$ Indices are raised and lowered using $g_{(0)}$.

${ }^{2}$ This is related with the on-shell value of the Einstein-Hilbert action for gravity with a negative cosmological constant as, $V_{A d S}=\frac{\kappa^{2}}{d} S_{A d S}^{o n-s h e l l}$, where $S_{A d S}=\frac{1}{2 \kappa^{2}} \int d^{d+1} x \sqrt{G}(R+2 \Lambda)$. Notice that we use the conventions in [7], in particular the curvature conventions are such that AdS has positive curvature.
} 
A free, massive scalar field $\Phi(x, r)$ in this $A d S$ background has action

$$
S=\frac{1}{2} \int d^{d+1} x \sqrt{G}\left(G^{\mu \nu} \partial_{\mu} \Phi \partial_{\nu} \Phi+M^{2} \Phi^{2}\right)
$$

with equation of motion (EOM) the Klein-Gordon equation with the Laplacian associated with $G_{\mu \nu}$. Via the usual AdS/CFT dictionary, this scalar will be dual to some gauge-invariant CFT operator with dimension $\Delta$ given by $M^{2}=(\Delta-d) \Delta$. A solution to the EOM resulting from the above action will, in general, have the form

$$
\begin{aligned}
\Phi(x, r) & =r^{d-\Delta} \phi(x, r) \\
& =r^{d-\Delta}\left(\phi_{(0)}+r \phi_{(1)}+\ldots+r^{2 \Delta-d} \phi_{(2 \Delta-d)}+r^{2 \Delta-d} \log \left(r^{2}\right) \psi_{(2 \Delta-d)}\right)+O\left(r^{\Delta+1}\right) .
\end{aligned}
$$

The logarithmic term appears only when $2 \Delta-d$ is an integer, which we will assume to be the case, and reflects the existence of matter conformal anomalies in the dual CFT, i.e. conformal anomalies due to short distance singularities in correlators of composite gauge invariant operators [16]. All coefficients $\phi_{(n)}$ for $n<2 \Delta-d$, as well as $\psi_{(2 \Delta-d)}$, can be computed recursively by inserting this form of the solution into the EOM and expanding in powers of $r$. The result is that the leading coefficient, $\phi_{(0)}$, and the coefficient $\phi_{(2 \Delta-d)}$ are not fixed by the EOM but all other coefficients are fixed by these two. The one coefficient we will need explicitly in terms of $\phi_{(0)}$ is the coefficient of the logarithmic term for the case $2 \Delta-d=2$ :

$$
\psi_{(2)}=-\frac{1}{2}\left[\square_{0} \phi_{(0)}+\left(\frac{d}{2}-1\right) \phi_{(0)} \operatorname{Tr} g_{(2)}\right] .
$$

The action naively evaluated on such a solution is again infinite because of IR divergences, so holographic renormalization proceeds as before. The resulting counterterms are given by

$$
\begin{aligned}
& L_{4}=\sqrt{\gamma} \frac{(d-\Delta)}{2}\left(1+\frac{1}{d-\Delta} \frac{1}{\log \epsilon}\right) \Phi^{2}(x, \epsilon) \\
& L_{5}=\frac{1}{2(2 \Delta-d-2)} \sqrt{\gamma}\left(\Phi(x, \epsilon) \square_{\gamma} \Phi(x, \epsilon)+\frac{d-\Delta}{2(d-1)} R[\gamma] \Phi(x, \epsilon)^{2}\right)
\end{aligned}
$$

These counterterms are the only ones required when $2 \Delta-d \leq 2$. The second term in $L_{4}$, proportional to $\frac{1}{\log (\epsilon)}$, only appears in our examples when the scalar saturates the BreitenlohnerFreedman (BF) bound for $A d S_{d+1}, M^{2} \geq-\frac{d^{2}}{4}$ [17], for which $2 \Delta-d=0$. In this case also $L_{4}$ alone suffices to renormalize the action. In the case that $2 \Delta-d=2$, the coefficient $[2(2 \Delta-d-2)]^{-1}$ in $L_{5}$ is replaced with $-\frac{1}{2} \log (\epsilon)[7]$.

Renormalized correlators in the boundary theory can be computed from the renormalized action. According to the AdS/CFT dictionary, the leading coefficient of the asymptotic expansion will act as a source for the dual operator. Denoting this operator as $O$, the onepoint function can be written

$$
\langle O\rangle=\frac{1}{\sqrt{g_{(0)}}} \frac{\delta S_{r e n}}{\delta \phi_{(0)}}
$$


which can in turn be written in terms of quantities on the $r=\epsilon$ slice:

$$
\langle O\rangle=\lim _{\epsilon \rightarrow 0}\left(\frac{1}{\epsilon^{\Delta}} \frac{1}{\sqrt{\gamma}} \frac{\delta S_{s u b}}{\delta \Phi(x, \epsilon)}\right) .
$$

For the cases we consider, if the scalar saturates the BF bound this will be modified: $\frac{1}{\epsilon^{\Delta}} \rightarrow$ $\frac{\log (\epsilon)}{\epsilon^{\triangle}}[17]$.

The structure of the counterterms and the renormalized one-point functions is most transparent in the radial Hamiltonian formalism, where the radius is playing the role of time $[18,19]$. This is also the fastest way to arrive at the results reviewed above, since in this method covariance is manifest at all stages. In this formalism the central object is the radial canonical momentum,

$$
\pi=\frac{\partial L}{\partial \dot{\Phi}}
$$

where overdot denotes differentiation w.r.t. the radius $\rho=-\log r$. It can be easily shown to correspond to a regularized one-point function,

$$
\langle O\rangle_{r e g} \equiv \frac{\delta S_{r e g}}{\delta \Phi(x, \epsilon)}=\pi(x, \epsilon) .
$$

This expression is infinite as the regulator is removed. To extract the finite part in a covariant fashion we use the fact that the theory possesses a well-defined dilatation operator,

$$
\delta_{D}=\int d^{d} x\left(2 \gamma_{i j} \frac{\delta}{\delta \gamma_{i j}}+(\Delta-d) \Phi \frac{\delta}{\delta \Phi}\right) .
$$

Let us expand the canonical momentum in eigenfunctions of $\delta_{D}$,

$$
\pi=\sqrt{\gamma}\left(\pi_{(d-\Delta)}+\cdots+\pi_{(\Delta)}+\tilde{\pi}_{(\Delta)} \log r^{2}+\cdots\right),
$$

where the subscripts indicate dilatation weight of the terms,

$$
\delta_{D} \pi_{(n)}=-n \pi_{(n)}, \quad \delta_{D} \tilde{\pi}_{(\Delta)}=-\Delta \tilde{\pi}_{(\Delta)}, \quad \delta_{D} \pi_{(\Delta)}=-\Delta \pi_{(\Delta)}-2 \tilde{\pi}_{(\Delta)} .
$$

Notice that the normalizable mode $\pi_{(\Delta)}$ transforms anomalously. This expansion is a covariant analogue of the radial expansion (2.7). The explicit form of $\pi_{(i)}$ is found by inserting (2.15) in Hamilton's equations and iteratively solving them by collecting terms with the same weight. This procedure determines all $\pi_{(i)}$ except $\pi_{(\Delta)}$ which is left undetermined, as in the discussion below (2.7).

The renormalized 1-point function is now simply given by the term of weight $\Delta$, as it should since the dual operator has dimension $\Delta$,

$$
\langle O\rangle=\pi_{(\Delta)}
$$

Furthermore, evaluating the regulating action on-shell one immediately gets

$$
S_{r e g}=-\int d^{d} x \sqrt{\gamma} \frac{1}{2} \pi \Phi
$$


and thus the counterterms are (minus) the terms involving the momentum eigenfunctions with weight less than $\Delta$ plus the logarithmic term involving $\tilde{\pi}_{\Delta}$. This is a general feature of the counterterm action for any theory, namely the counterterms are always certain linear combinations of momentum eigenfunctions with weight less than $\Delta$ plus logarithmic counterterms obtained by expanding the regulated action in eigenfunctions of the dilatation operator and using the basic relation (2.13) with $\delta \rightarrow \delta_{D}$,

$$
\delta_{D} S_{r e g}=\pi \delta_{D} \Phi+\pi^{i j} \delta_{D} \gamma_{i j}
$$

where we have reinstated the metric dependence. We refer to $[18,19]$ for the details and further discussion.

\section{Generalities for Probe Branes}

\subsection{General Solution and Counterterms}

All the cases we consider in this section are SUSY probe D-branes embedded in $A d S_{5} \times S^{5}$. In every case, the probe wraps some $A d S_{m} \times S^{n}$ with $|m-n|=2$ as required by supersymmetry [11]. We will focus on the cases with $m-n=2$. In all these cases, a single scalar field describes the embedding. These probe branes are thus described by a scalar in a fixed gravitational background, only with an action more complicated than that of a free scalar. In general the non-linear Born-Infeld action would lead to new counterterms. We will show, however, that in the class of embeddings we analyze the counterterms are identical to that of a free scalar. To show this it is sufficient to show that the leading asymptotic structure of the field equations (i.e. the orders where the normalizable and non-normalizable modes appear) is the same as that of the free scalar, and establish that the on-shell actions agree up to order $r^{m}$, the highest order at which divergences could appear in the action. From this follows (upon use of (2.13)) that the radial canonical momenta are the same up to $\pi_{(\Delta)}$ and thus the counterterm actions are the same. It also follows (and we will verify it explicitly) that the solution of the EOM has the same form as the free scalar solution to order $r^{\Delta}$.

In three of the four cases we consider, the scalar is a coordinate on the $S^{5}$ transverse to the brane. The $S^{n}$ is a trivial cycle on the $S^{5}$, so the scalar, which gives the position of the $S^{n}$ in a transverse direction, can have a profile such that the $S^{n}$ "slips off" the $S^{5}$ at some finite $r$. In the one exceptional case, for the D5 probe, the scalar is not a coordinate on the $S^{5}$ but is the position of the $A d S_{4}$ the brane wraps inside $A d S_{5}$. This case is not included in the analysis of this section.

The scalar "slipping mode" has mass-squared $M^{2}=-n$ (as we will show). Via the AdS/CFT dictionary the dimension of the dual operator is $\Delta=n$. In particular, this means that $d-\Delta=(m-1)-n=1$, so in every case we consider the scalar's asymptotic expansion will have a leading behavior of simply $r$. Recall also that the logarithmic term in the asymptotic expansion of the free scalar entered at order $r^{\Delta}$ which here will be $r^{n}$. The cases we consider are summarized in the following table of useful quantities: 


\begin{tabular}{|c|c|c|c|c|}
\hline Dp probe & $m=d+1$ & $n=\Delta$ & $2 \Delta-d$ & $-\frac{d^{2}}{4}$ \\
\hline D7 & 5 & 3 & 2 & -4 \\
\hline D5 & 4 & 2 & 1 & $-\frac{9}{2}$ \\
\hline D3 & 3 & 1 & 0 & -1 \\
\hline
\end{tabular}

Given appropriate coordinates on $A d S_{5}$, the metric of the $A d S_{m}$ subspace will have the same form as $(2.1)$ but now $\mu, \nu=0, \ldots, m-1$ and $i, j=0, \ldots, m-2$. Let the $S^{5}$ metric be

$$
d \Omega_{5}^{2}=d \Phi^{2}+\sin ^{2}(\Phi) d \theta^{2}+\cos ^{2}(\Phi) d \Omega_{3}^{2}
$$

with $d \Omega_{3}^{2}$ the standard metric on $S^{3}$. The $S^{n}$ 's with $n<3$ sit inside this $S^{3}$. The DBI action is then

$$
S=\int d^{m} x \sqrt{G} \cos ^{n}(\Phi) \sqrt{1+G^{\mu \nu} \partial_{\mu} \Phi \partial_{\nu} \Phi}
$$

where we neglect integration over the remaining $n$ variables because the cases we are interested in have no dependence on these coordinates. The resulting EOM for $\Phi$ is then

$$
0=\square \Phi+n \tan (\Phi)-\frac{1}{2} \frac{G^{\mu \nu} \partial_{\mu} \Phi \partial_{\nu}\left(G^{\rho \sigma} \partial_{\rho} \Phi \partial_{\sigma} \Phi\right)}{1+G^{\alpha \beta} \partial_{\alpha} \Phi \partial_{\beta} \Phi}
$$

where $\square$ is with respect to the $A d S_{m}$ metric. To order $r^{\Delta}$, this is identical to the EOM of a free scalar with $M^{2}=-n$ in a fixed $A d S_{m}$ background, which we explicitly show below. In the case of the free scalar the asymptotic solution has two undetermined coefficients, the source and the vev part, as we reviewed in section 2 . This matches the fact that the field equation is a second order linear differential equation in $r$, so two pieces of boundary data are required in order to specify the solution. This is also consistent with holography: on the field theory side the form of the Lagrangian and the specific vacuum we consider (the vev's) are the only information needed in order to specify the theory. We therefore expect that these facts will remain true for the asymptotic solution of the DBI field equation (3.3). Indeed, despite the higher derivative terms in the DBI action, the EOM (3.3) is still a second order differential equation in $r$ (now non-linear) and we now verify this expectation explicitly.

Consider a solution with the most general possible form:

$$
\Phi(x, r)=r\left[\sum_{i=0}^{\infty} \phi_{(i)} r^{i}+\sum_{j=0}^{\infty} \psi_{(j)} r^{j} \log (r)+\sum_{k=0}^{\infty} \sum_{l=2}^{p} \Psi_{(k, l)} r^{k} \log (r)^{l}\right] .
$$

We are allowing higher powers of $\log (r)$, but inserting this solution into the EOM shows that generically truncating the series at any finite order $p$ requires the $\Psi_{(k, l)}$ to all be zero. To see this, consider the leading term in the expansion of the EOM:

$$
\begin{aligned}
0 & =r\left[(m-3) \psi_{(0)}+2 \Psi_{(0,2)}+2 \log (r)\left((m-3) \Psi_{(0,2)}+3 \Psi_{(0,3)}\right)\right)+\ldots \\
& +(p-1) \log (r)^{p-2}\left((m-3) \Psi_{(0, p-1)}+p \Psi_{(0, p)}\right) \\
& \left.+p \log (r)^{p-1}(m-3) \Psi_{(0, p)}\right]+O\left(r^{2}\right)
\end{aligned}
$$


Clearly, for any finite $p, \Psi_{(0, p)}=0$ implies $\Psi_{(0, p-1)}=0$ and so on so that all $r \log (r)^{l}$ do not appear when $l \geq 2$. A similar story happens for $\Psi_{(1, p)}$, that is, all $r^{2} \log (r)^{l}$ are absent for $l \geq 2$. Of course, an infinite number of higher powers of $\log (r)$ could appear $(p=\infty)$, but this would invalidate the assumption that the solution has a power series expansion. Special cases arise when the numerical coefficients multiplying the highest power of $\log (r)$ are zero. For instance, $m=3$ is a special case in (3.5) for which the coefficient of $\log (r)^{p-1}$ is zero (although in this case one still obtains $\Psi_{(0, p)}=0$ ). These cases have to be analyzed separately and could result in higher powers of $\log (r)$ with coefficients determined in terms of the source and the vev coefficients. For $m=4,5$ we verified that higher powers of $\log (r)$ do not appear up to order $r^{5}$, that is, $\Psi_{(k, l)}=0$ for $k=2,3,4,5$. When $m=3, \Psi_{(2,2)}$ and $\Psi_{(2,3)}$ are non-zero but are determined in terms of $\psi_{(0)}$. Notice that such higher order logs also appear in the Coulomb branch example in [17] and that both examples with higher powers of logs are cases where the BF bound is saturated. It is unclear to us whether this is a general feature (i.e. that higher order logs appear only when the BF bound is saturated) or it is specific to the examples we considered.

What results from this discussion is that the form of the solution, up to the order we are interested in, is the same as that of a free scalar:

$$
\Phi(x, r)=r\left[\sum_{i=0}^{\Delta-1} \phi_{(i)} r^{i}+\sum_{j=0}^{\Delta-1} \psi_{(j)} r^{j} \log (r)\right]+O\left[r^{\Delta+1}\right] .
$$

Inserting this into the EOM gives, to order $r^{2}$,

$$
0=(m-3) \psi_{(0)} r+\left((m-4) \phi_{(1)}+(m-5+(m-4) \log (r)) \psi_{(1)}\right) r^{2}+O\left(r^{3}\right)
$$

To this order, this is identical to a free scalar in $A d S_{m}$ with mass-squared $M^{2}=-n$ and $d-\Delta=1$. In particular, the leading coefficient, $\phi_{(0)}(x)$ is left undetermined by the EOM, as expected: this will be a source or will contribute to the vev of the dual operator, depending on whether the scalar saturates the BF bound. Notice also that for all $m$ that we consider, $\psi_{(1)}$ is zero. For $m=3,4,(3.7)$ determines the form of solution up to order $r^{\Delta}$. The value $m=5$, however, requires the next order, $r^{3}$. When $m=5,(3.7)$ requires $\psi_{(0)}=\phi_{(1)}=0$ in which case the $r^{3}$ term is

$$
\begin{aligned}
0 & =r^{3}\left[\square_{0} \phi_{(0)}+\operatorname{Tr}\left(g_{(2)}\right) \phi_{(0)}+\frac{1}{3}(m-5) \phi_{(0)}^{3}-2(m-5) \phi_{(2)}\right. \\
& \left.+(7-m) \psi_{(2)}+2(5-m) \log (r) \psi_{(2)}\right]+O\left(r^{4}\right) .
\end{aligned}
$$

This shows that $\phi_{(2)}$ is undetermined and $\psi_{(2)}$ is fixed by $\phi_{(0)}$ in precisely the same way as the free scalar, (2.8), with $d=m-1=4$. This is a nontrivial result: for all values of $m$ we consider, the form of the solution is the same as that of a free scalar up to order $r^{\Delta}$.

Since the solution is the same up to order $r^{\Delta}$ so will the momentum dilatation eigenstates up to $\pi_{(\Delta)}$. It follows that the divergences will be the same as that of a free scalar, and we 
explicitely verify this now. Expanding the argument of (3.2) we obtain:

$$
\begin{aligned}
S & =\int d^{m} x \sqrt{G}\left[1+\frac{1}{2}\left[G^{\mu \nu} \partial_{\mu} \Phi \partial_{\nu} \Phi-n \Phi^{2}\right]\right. \\
& \left.+\left(-\frac{n}{12}+\frac{n^{2}}{8}\right) \Phi^{4}-\frac{1}{8}\left(G^{\mu \nu} \partial_{\mu} \Phi \partial_{\nu} \Phi\right)^{2}-\frac{n}{4} \Phi^{2} G^{\mu \nu} \partial_{\mu} \Phi \partial_{\nu} \Phi+O\left(r^{6}\right)\right] .
\end{aligned}
$$

First notice that the leading term is simply the volume of $A d S_{m}$, requiring the counterterms $L_{1}, L_{2}$ and $L_{3}$ of the last section.

The leading $\Phi$ dependence is that of a free scalar in a fixed $A d S_{m}$ background with $M^{2}=-n$, as advertised. As $\sqrt{G}=\frac{1}{r^{m}} \sqrt{g}$ and we care only about $m \leq 5$, all terms of order $r^{6}$ or higher cannot contribute to divergences. When $m<5$ the $\Phi^{4}$ terms, which go at leading order as $r^{4}$, will not produce divergences, and hence in these cases the free scalar counterterms $L_{4}$ and $L_{5}$ are sufficient. When $m=5$ these terms may produce logarithmic divergences, but these terms cancel against each other to order $r^{4}$ because $n=3$ :

$$
\left(-\frac{n}{12}+\frac{n^{2}}{8}\right) \Phi^{4}-\frac{1}{8}\left(G^{\mu \nu} \partial_{\mu} \Phi \partial_{\nu} \Phi\right)^{2}-\frac{n}{4} \Phi^{2} G^{\mu \nu} \partial_{\mu} \Phi \partial_{\nu} \Phi=\left[\left(-\frac{n}{12}+\frac{n^{2}}{8}\right)-\frac{1}{8}-\frac{n}{4}\right] \phi_{(0)}^{4} r^{4}+O\left(r^{6}\right)
$$

\subsection{Supersymmetric Background Solution}

In the three cases with a scalar describing the slipping mode it is easy to check that with $g_{i j}(x, r)=\eta_{i j}$ an exact solution to the EOM preserving half the supersymmetries is

$$
\Phi=\arcsin (c r)=c r+\frac{c^{3}}{6} r^{3}+\ldots,
$$

that is, a solution of the form (3.6) with $\phi_{(0)}=c, \phi_{(1)}=0, \phi_{(2)}=\frac{c^{3}}{6}$ and no logarithmic terms. This solution describes a D-brane extending up to

$$
r_{\max }=\frac{1}{c} \text {. }
$$

$c$ sets the mass of a fundamental matter field in the dual field theory. In the flat embedding space this embedding just describes a planar D-brane locate at a fixed distance away from the stack of D3-branes,

$$
X=\frac{1}{r} \sin (\Phi)=c
$$

where $X$ is one of the flat embedding space coordinates written in a spherical coordinate system.

We will be mostly interested in calculating correlation functions, in particular the onepoint function, in this particular background. In order to ensure that holographic renormalization gives finite answers for all correlation functions we need to make sure however to add all the counterterms for arbitrary boundary values of the fields, so that we get finite answers for expectation values in the presence of sources. While the counterterms and the background solution are very similar in all the cases, the physical interpretation as well as the details of the procedure are quite different in all the cases and hence we will turn to the examples one by one. 


\section{The D3/D7 System}

\subsection{The System}

In the supersymmetric embedding of a probe D7 in $A d S_{5} \times S^{5}$, the D7 fills the $5 \mathrm{D}$ spacetime and wraps an $S^{3}$ inside the internal space, that is, the probe wraps an $A d S_{5} \times S^{3}$ and hence $m=5, n=3$, and the scalar's mass is above the BF bound. This system was proposed in [9] as a way to introduce flavor, that is fundamental representations, into the AdS/CFT correspondence. The particular supersymmetric case we are studying is dual to the $\mathcal{N}=4$ SYM theory coupled to a fundamental hypermultiplet that preserves $\mathcal{N}=2$ supersymmetry. Already in [9] it was noted that the gravity side has a non-zero subleading term $\phi_{(2)}$ in its asymptotic expansion, even though from the field theory side it is clear that no vev is allowed. Some arguments were given that on the gravity side the vev really is zero. Starting from [20] it has become common practice to read off the vev from the subleading term in the flat embedding space coordinate $X=\frac{1}{r} \sin (\Phi)$ which on the background solution is constant and indeed has no subleading term. The absence of any rigorous derivation of the correct procedure to calculate the vev became apparent with the work of [21] where different answers were found depending on what coordinate system was used. In their work a very natural choice was again "apparent" that yielded the expected answers, but the question arose how one would find the right coordinate system in less symmetric cases. Here we will apply holographic renormalization to that problem and will find an unambiguous answer.

\subsection{Counterterms}

In addition to the counterterms we derived for general $m$ and $n$ above, only in the case of the D7 brane we face an interesting new subtlety: the possibility of finite counterterms. A counterterm of the form

$$
L_{f}=\alpha \sqrt{\gamma} \Phi(x, \epsilon)^{4}
$$

will not introduce divergences for any $\alpha$, but will change the on-shell action by a finite amount depending on the free coefficient $\alpha$. Different values of $\alpha$ correspond to different renormalization schemes and some correlation functions will contain scheme-dependent terms. As first pointed out in [17] in the special case that one is interested in correlation functions in a supersymmetric background, one can fix the finite counterterms by picking the unique scheme in which supersymmetry is preserved. In the supersymmetric scheme not just the divergent pieces of the on-shell action but the action as a whole has to vanish when calculated on the supersymmetric background solution. Otherwise the ground state energy in the dual field theory would not be zero and hence supersymmetry would be broken.

For the D7 case the counterterms read

$$
\begin{aligned}
& L_{1}=-\frac{1}{4} \sqrt{\gamma} \\
& L_{2}=-\frac{1}{48} \sqrt{\gamma} R_{\gamma}
\end{aligned}
$$




$$
\begin{aligned}
L_{3} & =-\log \epsilon \sqrt{\gamma} \frac{1}{32}\left(R_{i j} R^{i j}-\frac{1}{3} R_{\gamma}^{2}\right) \\
L_{4} & =\frac{1}{2} \sqrt{\gamma} \Phi^{2}(x, \epsilon) \\
L_{5} & =-\frac{1}{2} \log (\epsilon) \sqrt{\gamma} \Phi(x, \epsilon)\left(\square_{\gamma}+\frac{1}{6} R_{\gamma}\right) \Phi(x, \epsilon)
\end{aligned}
$$

For the supersymmetric embedding, $g_{i j}(x, r)=\eta_{i j}$ and the curvature on the slice is zero so only $L_{1}$ and $L_{4}$ contribute. To fix the finite counterterm we only need to plug the background solution (3.11) into the action. On the background solution the action exactly reduces to

$$
S_{\text {reg }}=\int d^{4} x \int_{\epsilon}^{r_{\max }} d r \frac{1}{r^{5}}\left(1-c^{2} r^{2}\right)=\int d^{4} x\left(-\frac{1}{4} \frac{1}{r^{4}}+\left.\frac{1}{2} \frac{c^{2}}{r^{2}}\right|_{\epsilon} ^{r_{\max }}\right) .
$$

Indeed the action picks up a non-vanishing contribution of $-\frac{1}{4 r_{\max }^{4}}+\frac{c^{2}}{r_{\max }^{2}}=+\frac{c^{4}}{4}$ from the IR boundary. The UV divergence is ensured to cancel by the counterterms we introduced above. $L_{4}$ gives an additional finite piece, $L_{4}=\ldots+\frac{c^{4}}{6}+\ldots$. All in all we see that in order to set the on-shell action to zero we need

$$
\alpha=-\frac{5}{12}
$$

\subsection{One-point function}

Holographic renormalization tells us that the vev is obtained as the $\epsilon \rightarrow 0$ limit of

$$
\langle O\rangle=\frac{1}{\epsilon^{3} \sqrt{\gamma}} \frac{\delta S_{s u b}}{\delta \Phi(\epsilon)}
$$

In this case,

$$
\frac{\delta S_{\text {reg }}}{\delta \Phi}=\left.\frac{\delta L}{\delta \Phi^{\prime}}\right|_{\epsilon}=-\frac{1}{\epsilon^{3}} \sqrt{g} \cos ^{3}(\Phi(\epsilon)) \frac{\Phi^{\prime}(\epsilon)}{\sqrt{1+\epsilon^{2} \Phi^{\prime}(\epsilon)^{2}}} .
$$

This then contributes divergent and finite parts to the vev

$$
\langle O\rangle_{\text {reg }}=-\frac{\phi_{(0)}}{\epsilon^{2}}-3 \psi_{(2)} \log (\epsilon)+\left(2 \phi_{(0)}^{3}-3 \phi_{(2)}-\psi_{(2)}\right)+O\left(\epsilon^{2}\right)
$$

Adding in addition the variations of the counterterms we finally obtain for the vev

$$
\langle O\rangle=\log (\epsilon)\left(-2 \psi_{(2)}-\frac{1}{6} \phi_{(0)} R_{0}-\square_{0} \phi_{(0)}\right)+\left(\frac{1}{3} \phi_{(0)}^{3}-2 \phi_{(2)}-\psi_{(2)}\right)+O\left(\epsilon^{2}\right) .
$$

The coefficient of the $\log (\epsilon)$ is zero via the EOM, (2.8), and inserting the value of $\psi_{(2)}$ from (2.8) into the finite piece then gives

$$
\langle O\rangle=-2 \phi_{(2)}+\frac{\phi_{(0)}^{3}}{3}+\frac{R_{0}}{12} \phi_{(0)}+\square_{0} \phi_{(0)}
$$


The last two terms come from the curvature of the induced metric on the $r=\epsilon$ slice and can in fact be eliminated by adding a finite counterterm proportional to the matter conformal anomaly. On the background solution $\sin (\Phi)=c r$ we have $\phi_{(0)}=c$ and $\phi_{(2)}=\frac{c^{3}}{6}$, so indeed

$$
\langle O\rangle_{\text {background }}=0 \text {. }
$$

The finite counterterms required by supersymmetry set the vev to zero despite the appearance of a non-vanishing subleading term in the asymptotic expansion of the scalar field.

\section{The D3/D5 System}

\subsection{The System}

In the supersymmetric embedding of a probe D5 in $A d S_{5} \times S^{5}$, the D5 fills an $\mathrm{AdS}_{4}$ subspace of the 5D spacetime and wraps an $S^{2}$ inside the internal space, that is, the probe wraps an $A d S_{4} \times S^{2}$ and hence $m=4, n=2$ and the scalar's mass is above the BF bound. This system was introduced in [10] and analyzed in detail in [22]. The dual field theory in this case is a defect conformal field theory. Again the probe brane in the bulk corresponds to the introduction of fundamental matter in the field theory, but this time the matter only lives on a $3 \mathrm{~d}$ defect in the $4 \mathrm{~d}$ field theory. While the defect breaks translation invariance in the directions orthogonal to the defect, the field theory remains conformal as long as the matter is massless. The subgroup of the full conformal group that is preserved is the $S O(3,2)$ that leaves the position of the defect invariant. This symmetry in the bulk demands the worldvolume to be $\mathrm{AdS}_{4}$. The supersymmetric background solution once more describes adding a mass term to the fundamental matter. A new possibility that arises in this case is to turn on the scalar that corresponds to the embedding of the $\mathrm{AdS}_{4}$ inside the $\mathrm{AdS}_{5}$ [11]. This deformation breaks all supersymmetries. On the field theory side it was argued in [11] that one turned on a vacuum expectation value for the defect field, as we will verify explicitly below. We will also see that the energy of the configuration in non-zero. On the field theory side one certainly expects such configurations to eventually relax to the supersymmetric ground state. On the gravity side, this process should be mapped to some instability of the brane embedding that drives the vev to zero. It would be interesting to analyze the stability of this embedding. This would provide an example of an unstable (or perhaps metastable) configurations that exists and can be studied both at weak and strong coupling.

\subsection{Mass deformation}

In order to cancel the UV divergences we need to add the following counterterms:

$$
\begin{aligned}
L_{1} & =-\frac{1}{3} \sqrt{\gamma} \\
L_{2} & =-\frac{1}{12} \sqrt{\gamma} R_{\gamma} \\
L_{4} & =\frac{1}{2} \sqrt{\gamma} \Phi^{2}(x, \epsilon)
\end{aligned}
$$




$$
L_{5}=-\frac{1}{2} \sqrt{\gamma} \Phi(x, \epsilon)\left(\square_{\gamma}+\frac{1}{4} R_{\gamma}\right) \Phi(x, \epsilon)
$$

No option to add finite counterterms involving only $\Phi$ and $\sqrt{\gamma}$ arises. Indeed with this set of counterterms the on-shell action on the background solution is zero already,

$$
S_{r e g}=\int d^{3} x \int_{\epsilon}^{r_{\max }} d r \frac{1}{r^{4}} \sqrt{1-c^{2} r^{2}}=\int d^{3} x\left(-\left.\frac{\left(1-c^{2} r^{2}\right)^{3 / 2}}{3 r^{3}}\right|_{\epsilon} ^{r_{\max }}\right) .
$$

The IR term, with $r_{\max }=\frac{1}{c}$, is identically zero.

With these counterterms it is again straightforward to calculate the vev. Setting to zero the terms involving $R_{\gamma}$ and $\square_{\gamma}$ one simply obtains

$$
\langle O\rangle=-\phi_{(1)} .
$$

Again, the supersymmetric background solution which has $\phi_{(1)}=0$ has no vev, as expected.

\subsection{Vev deformation}

In this subsection we study the probe RG flow obtained by turning on a scalar that controls the embedding of the $\mathrm{AdS}_{4}$ part inside the $\mathrm{AdS}_{5}$ while keeping the brane wrapped on the maximal sphere inside $S^{5}, \Psi=0$ [11]. For simplicity in this case we will only calculate the vev on the background solution; no problems are anticipated in once more working out the full holographic RG program for arbitrary correlation functions. Looking for an embedding $x_{3}=x(r)$ the induced metric on the $4 \mathrm{~d}$ part becomes

$$
d s^{2}=\frac{1}{r^{2}}\left(-d t^{2}+d x_{1}^{2}+d x_{2}^{2}+\left(1+\left(x^{\prime}\right)^{2}\right) d r^{2}\right)
$$

and the corresponding action is

$$
S=\int \frac{d^{4} x}{r^{4}} \sqrt{1+\left(x^{\prime}\right)^{2}}
$$

The most general solution to the equations of motion with the ansatz that $x_{3}$ only depends on $r$ is

$$
x^{\prime}=\frac{c r^{4}}{\sqrt{1-c^{2} r^{8}}},
$$

so that asymptotically

$$
x=r_{0}+\frac{c}{5} r^{5}+\ldots
$$

This brane extends up to

$$
r_{\max }=\frac{1}{c^{1 / 4}} .
$$

The solution with $c=0$ is the supersymmetric $\mathrm{AdS}_{4} \times S^{2}$ defect. What happens at $r=r_{\max }$ for the vev deformation is quite distinct from what we have encountered in the other examples. While before a sphere was shrinking in the internal space so that the brane smoothly 
terminates, for the vev deformation the internal sphere has constant size. To understand the physics of this embedding it is useful to note that close to $r_{\max }$ we simply get

$$
x^{\prime}=\frac{\sqrt{r_{\max }}}{2 \sqrt{2}} \frac{1}{\sqrt{r_{\max }-r}}+\ldots, \quad x=\sqrt{\frac{r_{\max }}{2}} \sqrt{r_{\max }-r}+\ldots
$$

Instead of ending at $r=r_{\max }$ the string turns around and hits the boundary again. The probe brane no longer is dual to the defect conformal field theory of a single D5 brane defect, but with a D5 and anti-D5 defect seperated by a distance $d=2 \frac{\sqrt{\pi} \Gamma(13 / 8)}{5 \Gamma(9 / 8)} r_{\max }$.

The on-shell action can be evaluated exactly

$$
S_{r e g}=\int d^{3} x \int_{\epsilon}^{r_{\max }} d r \frac{1}{r^{4}\left(1-c^{2} r^{2}\right)}=\int d^{3} x\left(\frac{1}{3 \epsilon^{3}}-c^{3 / 4} \frac{\sqrt{\pi} \Gamma(13 / 8)}{15 \Gamma(9 / 8)}+O(\epsilon)\right)
$$

where the finite term comes from the IR boundary. It follows that we should add to the action the counterterm

$$
L_{1}=-\frac{1}{3} \sqrt{\gamma}
$$

With this counterterms the action is not just finite but zero for the supersymmetric embedding, and is negative for the non-supersymmetric embedding. For the general $x(r)$ solution we get for the vev

$$
\langle O\rangle=\frac{1}{\epsilon^{3}} \frac{1}{\sqrt{\gamma}} \frac{\delta S_{s u b}}{\delta x}=-\left.\frac{1}{\epsilon^{4}} \frac{x^{\prime}}{\sqrt{1+\left(x^{\prime}\right)^{2}}}\right|_{\epsilon}=-c .
$$

This agrees with the expectation that a non-vanishing $c$ corresponds to a vev-induced flow once one realizes that $\frac{x}{r}$ is dual to an operator of dimension four [22]. The negative energy does not signal an instability of the original D5 brane defect since, as we noted above, the vev deformation really is dual to a system with 2 defects, one due to a D5 and one due to an anti-D5. It is not too surprising that latter system can lower its energy by moving out onto the Higgs branch. Since the constant $c$ is completely fixed by the distance $d$ between the defects we expect this configuration to correspond to the stable ground state of the system.

\section{The D3/D3 System}

\subsection{The System}

The D3/D3 system is another example that can be treated with the same methods. In this case, the probe D3's wrap an $A d S_{3} \times S^{1}$ inside $A d S_{5} \times S^{5}, m=3$ and $n=1$. This system was analyzed in [12]. It corresponds once more to a defect conformal field theory, this time with the fundamental hypermultiplet localized on a $2 \mathrm{~d}$ defect in the $4 \mathrm{~d}$ field theory. The interesting new aspect of this system is that the mass of the slipping mode saturates the BF bound. This gives rise to a slightly different structure of counterterms in holographic renormalization; it also leads to an interesting reversal in the role of the vev versus mass as we will see below.

From the boundary theory point of view it is also clear that this system is different. In all the other cases we had a higher dimensional brane intersecting the D3 brane, so that in the 
field theory limit the worldvolume fields on the higher dimensional brane decoupled together with gravity. The only dynamical fields remaining were those on the D3 branes and on the intersection from the 3-7 or 3-5 strings respectively. For the D3/D3 system the $\mathcal{N}=4 \mathrm{SYM}$ on both D3 stacks remains dynamical, so the field theory is really the theory of two $\mathcal{N}=4$ theories coupled to each other via a bifundamental hypermultiplet living on a $2 \mathrm{~d}$ defect. In particular, the spacetime separation between the two stacks of D3 branes can be viewed as a vev for one of the additional D3's worldvolume fields and should no longer be interpreted as a parameter (as in the D5 or D7) case, but as a vev of a dynamical field. We will see that holographic renormalization correctly accounts for this effect. It is precisely separating the two stacks in spacetime that is described by the supersymmetric background solution we studied for general $m$ and $n$, and we will see that unlike in the other cases for $m=3, n=1$ it is a vev instead of a mass deformation. Implementing holographic renormalization properly we also calculate the two-point function in this case, yielding a different answer from the one originally advertised in [12].

\subsection{Counterterms and One-Point Function}

The interesting change in roles between source and vev can already be observed from the form of the asymptotic solution which in this case reads

$$
\Phi=r\left(\tilde{\psi}_{(0)}+\psi_{(0)} \log (r)+\ldots\right)
$$

for which the first term is normalizable and will set the vev, while the second term is nonnormalizable and will correspond to a source in the dual theory. On our supersymmetric background solution the source term is zero; we will calculate the vev momentarily. For $m=3, n=1$ the counterterms become

$$
\begin{aligned}
& L_{1}=-\frac{1}{2} \sqrt{\gamma} \\
& L_{2}=\frac{1}{4} \log \epsilon \sqrt{\gamma} R_{\gamma} \\
& L_{4}=\frac{1}{2} \sqrt{\gamma} \Phi^{2}(x, \epsilon)\left(1+\frac{1}{\log \epsilon}\right)
\end{aligned}
$$

No finite counterterms can arise and it is again easy to verify that the on-shell action for our supersymmetric background vanishes with these counterterms already.

In this case, the vev is the $\epsilon \rightarrow 0$ limit of

$$
\langle O\rangle=\frac{\log (\epsilon)}{\epsilon} \frac{1}{\sqrt{\gamma}} \frac{\delta S_{\text {sub }}}{\delta \Phi(\epsilon)} .
$$

Using the counterterms it is again straightforward to obtain

$$
\langle O\rangle=\tilde{\psi}_{(0)}
$$

where we again set to zero the terms involving the curvature on the slice. The vev is thus indeed determined by the coefficient of the normalizable mode; on our supersymmetric background we simply have $\langle O\rangle=c$. As expected, the brane separation shows up as a vev and no longer as a mass term. 


\subsection{Two-point functions}

We discuss in this section the computation of correlation functions. $n$-point functions can be obtained from the exact one-point function by further differentiating $(n-1)$ times w.r.t. the source. It follows that in order to obtain the two-point function it is sufficient to solve the linearized equation of motion.

The simplest case to consider is the two-point function for the theory specified by the embedding $\Phi=0$. We first discuss the 2-point function of the operator dual to the scalar field $\Phi$. This can be obtained by linearizing the field equation (3.3) around $\Phi=0$, and Fourier transforming in the spatial directions we get

$$
r^{2} \Phi^{\prime \prime}-r \Phi^{\prime}-\left(k^{2} r^{2}-1\right) \Phi=0
$$

This is equal (as it should be) to the linearization of the field equations of a scalar of mass $M^{2}=-1$. The solution of this equation that is regular in the interior is

$$
\Phi(r, k)=r K_{0}(k r) .
$$

Expanding for small $r$ we get

$$
\Phi(r, k)=\psi_{(0)}(k) r\left(\log r+\frac{1}{2} \log \frac{k^{2}}{\mu^{2}}\right)
$$

where $\psi_{(0)}(k)$ represents the overall normalization of the solution, and $\mu^{2}=4 e^{-2 \gamma}$ ( $\gamma$ is the Euler constant). Proper units are restored using the AdS radius. It follows that

$$
\begin{gathered}
\tilde{\psi}_{(0)}(k)=\psi_{(0)}(k) \frac{1}{2} \log \frac{k^{2}}{\mu^{2}} \\
\langle O(k) O(-k)\rangle=-\frac{\delta \tilde{\psi}_{(0)}}{\delta \psi_{(0)}}=-\frac{1}{2} \log \frac{k^{2}}{\mu^{2}}
\end{gathered}
$$

Fourier transforming we get the renormalized version of $1 / x^{2}$ (see appendix)

$$
\langle O(x) O(0)\rangle=\frac{1}{2 \pi}\left(\frac{1}{x^{2}}\right)_{R}
$$

(with $m=1$ ), as is appropriate for the two-point function for a scalar operator of dimension one.

We now discuss the computation of 2-point function which are dual to fields parametrizing the flunctuations of $A d S_{3}$ in $A d S_{5}$. The mass of the fluctuations is [12],

$$
m^{2}=(l-1)(l-3)
$$

with $l$ a positive integer. One should distiguish between the fluctuations with mass

$$
m^{2}>-\frac{d^{2}}{4}+1=0 \quad \Rightarrow \quad l>3
$$


in which case there is a single branch of dual operators with dimension,

$$
\Delta=l-1, \quad l>3
$$

and fluctuations with mass

$$
-\frac{d^{2}}{4}=-1 \leq m^{2} \leq-\frac{d^{2}}{4}+1=0, \quad \Rightarrow \quad 1 \leq l \leq 3
$$

In this case there are two inequivalent quantizations and we have two branches of operators. The $l=2$ correspond to $m^{2}=-1$, so the fluctuations saturate the BF bound. We discussed the computation of the 2-point functions for this case in the beginning of this section. The $l=1$ and $l=3$ cases correspond to massless fluctuations. The $l=1$ case is special: the corresponding boundary operator has dimension zero and thus saturates the unitarity bound. From the bulk point of view this case utilizes the $\Delta_{-}$branch and the roles of source and vev are exchanged. The computation of correlation functions for such cases has been discussed in [25]. When the operators saturate the unitarity bound, however, the normalization of the (holographically computed) 2-point function turns out to vanish (set $\Delta=d / 2-1$ in (2.19) or (2.21) of [25]) even though the corresponding QFT 2-point function is generically non-zero indicating subtleties with the normalization of these operators. Leaving this special case aside we now discuss the computation of 2 -point functions for $l \geq 3$.

In our analysis so far we have constructed the counterterms required to render the onshell action finite and then derived the 1-point function. This can also be done for the case at hand and this would uniquely fix (up to finite scheme dependent terms) all boundary terms. We should also note that another (perhaps more fundamental) requirement on the action is that it is stationary when the field equations hold and appropriate boundary conditions are imposed. This also fixes all boundary terms to be the ones determined by finiteness [23].

In this section we are only interested in computing 2-point functions, however, so instead of first computing the counterterms that would render finite any correlation function we will follow the simpler route developed in [19]. 2-point functions are determined by solving the linearized fluctuation equations and to renormalize we need to perform a near-boundary analysis of the fluctuation equations.

The fluctuation equations can be obtained from (3.16) of $[12]^{3}$. In our coordinates these read

$$
r^{2} \tilde{w}_{l}^{\prime \prime}-r \tilde{w}_{l}^{\prime}-\left(k^{2} r^{2}+m^{2}\right) \tilde{w}_{l}=0
$$

where we Fourier transformed along the boundary directions. In the recent work [24] the fluctuation equations were analyzed for D3 branes separated by a distance $L$ and shown to be of the hypergeometric type. One can thus readily derive the 2-point functions in this more general setting but for simplicity we restrict to the $L=0$ case. The fluctuation equation in (6.17) can also be obtained from (4.10) of [24] in the limit $L \rightarrow 0$. The solution of (6.17) that is regular in the interior is

$$
\tilde{w}_{l}(r, k)=r K_{(l-2)}(k r)
$$

\footnotetext{
${ }^{3}$ We employ the $\tilde{w}$ notation of [12], whereas [24] uses $w$. The two are related by $w=r \tilde{w}$
} 
We now discuss how to extract renormalized 2-point functions from the solution of the fluctuation equation. We will follow the Hamiltonian formalism described in the introduction. It is useful to introduce a new radial coordinate $\rho=-\log r$. In these coordinates the $A d S_{3}$ metric reads

$$
d s^{2}=d \rho^{2}+e^{2 \rho} d x^{i} d x^{i}
$$

and the canonical momenta are given by ${ }^{4}$

$$
\pi_{l}=\dot{\tilde{w}}_{l}
$$

where overdot denotes differentiation w.r.t. $\rho$. The fluctuation equation (6.17) becomes

$$
\dot{\pi}_{l}+2 \pi_{l}-\left(\xi+m^{2}\right) \tilde{w}_{l}=0
$$

where we defined $\xi=e^{-2 \rho} k^{2}$, which comes from the Fourier transform of $\square_{\gamma}$ at the regulating surface $r=$ const and $\gamma_{i j}=e^{2 \rho} \delta_{i j}$ is the corresponding induced metric.

Since we are interested in 2-point functions, we need to know the radial canonical momentum as a function of the induced field to linear order. Covariance fixes their relation to be the form

$$
\pi_{l}=f_{l}(\xi) \tilde{w}_{l}
$$

The regularized 2-point function (with the regulator being a small constant value of the radial coordinate $r=\epsilon$ ) is then given by

$$
\begin{aligned}
\left\langle O_{l-1}(k) O_{l-1}(-k)\right\rangle_{\epsilon} & =-\frac{1}{\epsilon^{2(l-2)}} f_{l}(\xi) \\
& =-\frac{1}{\epsilon^{2(l-2)}}\left((l-3)+k \epsilon \frac{K_{(l-3)}(k \epsilon)}{K_{(l-2)}(k \epsilon)}\right)
\end{aligned}
$$

In the limit $\epsilon \rightarrow 0$ these correlators diverge. Our task is to extract correctly the finite part.

As reviewed in section 2, the renormalized 1-point function in the presence of sources is given by the part of the canonical momentum with dilatation weight equal to the dimension of the dual operator $[18,19]$. To extract this part we need the asymptotic form of $f_{l}(\xi)$. To this end, we insert (6.22) in (6.21) to obtain

$$
\dot{f}_{l}+f_{l}^{2}+2 f_{l}-\left(\xi+m^{2}\right)=0
$$

From the general discussion in the introduction we know that the $f_{l}(\xi)$ has an expansion of the form,

$$
f_{l}(\xi)=f_{l(0)}+f_{l(2)}+\cdots+f_{l(2 l-4)}+\tilde{f}_{l(2 l-4)} \log r^{2}+\cdots
$$

Furthermore, in our case the dilatation operator in (2.14) is just equal to the radial derivative,

$$
\delta_{D}=\partial_{\rho}
$$

\footnotetext{
${ }^{4}$ Strictly speaking, the momenta are densities and one should include a factor of $\sqrt{\gamma}$ as in the formulae in the introduction. The equations are simpler without these factors, however, so we will work with (6.20).
} 
This follows from the fact that the background is $A d S_{3}$ (insert $\gamma_{i j}=e^{2 \rho} \delta_{i j}, \Phi=0$ in (2.14)).

Inserting the expansion (6.25) into (6.24) and organizing the terms according to their dimension one obtains all coefficients except for $f_{l(2 l-4)}$ which is left undetermined. Let us explain this computation. In (6.24) the mass term has dimension 0 and $\xi$ has dimension 2, so $f_{l(0)}$ and $f_{l(2)}$ are different from the remaining $f_{l(2 p)}$. We get

$$
\begin{aligned}
& l \neq 3: \quad f_{l(0)}=l-3, \quad f_{l(2)}=\frac{\xi}{2(l-3)} \\
& l=3: \quad f_{3(2)} \text { undetermined, } \quad \tilde{f}_{3(2)}=-\frac{\xi}{2}
\end{aligned}
$$

For the remaining coefficients one gets a recursive relation,

$$
\begin{aligned}
& p \neq l-2: \quad f_{l(2 p)}=\frac{1}{2(p-l+2)} \sum_{n=1}^{p-1} f_{l(2(p-n))} f_{l(2 n)} \\
& p=l-2: \quad f_{l(2 l-4)} \text { undetermined, } \quad \tilde{f}_{l(2 l-4)}=\frac{1}{2} \sum_{n=1}^{l-3} f_{l(2(l-2-n))} f_{l(2 n)}
\end{aligned}
$$

Having determined the asymptotic expansion we now extract the renormalized 2-point function as

$$
\left\langle O_{l-1}(k) O_{l-1}(-k)\right\rangle=-\lim _{\epsilon \rightarrow 0} \frac{1}{\epsilon^{2(l-2)}}\left((l-3)+k \epsilon \frac{K_{(l-3)}(k \epsilon)}{K_{(l-2)}(k \epsilon)}-\sum_{p=0}^{l-3} f_{l(2 p)}-\tilde{f}_{l(2 l-4)} \log \epsilon^{2}\right)
$$

Let us work out explicitly the first few cases $\left(\mu^{2}=4 e^{-2 \gamma}\right.$ as before):

$$
\begin{aligned}
& l=3:\left\langle O_{2}(k) O_{2}(-k)\right\rangle=\frac{1}{2} k^{2} \log \frac{k^{2}}{\mu^{2}} \\
& l=4:\left\langle O_{3}(k) O_{3}(-k)\right\rangle=-\frac{1}{8}\left(k^{2}\right)^{2} \log \frac{k^{2}}{\mu^{2}} \\
& l=5: \quad\left\langle O_{4}(k) O_{4}(-k)\right\rangle=\frac{1}{128}\left(k^{2}\right)^{3}\left(\log \frac{k^{2}}{\mu^{2}}-\frac{1}{2}\right)
\end{aligned}
$$

which is the correct behavior for two-point operator of dimension 2, 3 and 4, respectively. (The last term in the r.h.s. of the $l=5$ case is scheme dependent. It contributes a contact term in $x$ space). Fourier transforming to $x$ space (using the results of the appendix) we find

$$
\left\langle O_{\Delta}(x) O_{\Delta}(0)\right\rangle=\frac{2 \nu \Gamma\left(\frac{d}{2}+\nu\right)}{\pi^{d / 2} \Gamma(\nu)}\left(\frac{1}{x^{2 \Delta}}\right)_{R}
$$

where the subscript $R$ indicates that the expression is renormalized and $\nu=\Delta-d / 2$. The normalization of the 2-point functions coincides with the one determined in [26] (as it should). 
By construction, the subtractions in (6.29) can be implemented by means of counterterms. The explicit form follows from (2.18) and is given by

$$
\begin{aligned}
S_{c t} & =\frac{1}{4} \sum_{l \geq 3} \int d^{2} x \sqrt{\gamma} \tilde{w}_{l}\left(\sum_{p=0}^{l-3} f_{l(2 p)}+\log \epsilon^{2} \tilde{f}_{l(2 l-4)}\right) \tilde{w}_{l}^{*}+c . c . \\
& =\frac{1}{4} \sum_{l \geq 3} \int d^{2} x \sqrt{\gamma}\left((l-3) \tilde{w}_{l} \tilde{w}_{l}^{*}-\frac{1}{2(l-3)} \tilde{w}_{l} \square_{\gamma} \tilde{w}_{l}^{*}+\cdots\right)+c . c .,
\end{aligned}
$$

(when $l=3$ there is only the logarithmic counterterm). The renormalized action is given by the sum of the bulk action and this boundary term. The action derived in [12] should be viewed as a "bare" action. Adding to it appropriate boundary terms leads to a renormalized action and the corresponding renormalized correlators exhibit power law behavior.

The fields discussed here are dual to supersymmetric operators that saturate a BPS bound [12]. A realization of these operators in terms of defect fields has been proposed in [12] and they contain massless scalar fields. As discussed there this implies that in general there will be strong infrared effects. The holographic computation discussed here and the fact that these operators are supersymmetric suggest that appropriate IR renormalization would lead to 2-point functions exhibiting power law behavior at weak coupling. We leave the investigation of this issue for future work.

\section{Appendix: Fourier Transform}

In differential regularization [27], $1 / x^{2}$ is represented by

$$
\left(\frac{1}{x^{2}}\right)_{R}=\frac{1}{8} \square \log ^{2} x^{2} m^{2}
$$

where the subscript $R$ indicates that this is a renormalized expression and $m^{2}$ is the renormalization scale. The renormalized expression differs from $1 / x^{2}$ by the infinite term $\delta(x) \log x^{2} m^{2}$ localized at $x=0$.

We now compute the Fourier transform following [28]. Integrating by parts once and after some manipulations one arrives at

$$
\begin{aligned}
I(k) & =\int d^{2} x e^{i k x} \frac{1}{8} \square \log ^{2} x^{2} m^{2}=-i \frac{k_{\mu}}{2} \int d^{2} x e^{i k x} \frac{x^{\mu}}{x^{2}} \log x^{2} m^{2} \\
& =-\left.\pi \frac{d}{d a}\left(\left(\frac{k^{2}}{\mu^{2}}\right)^{a} F(a)\right)\right|_{a=0}
\end{aligned}
$$

where $\mu^{2}=4 e^{-2 \gamma} m^{2}$ and

$$
F(a)=-a \frac{2^{2 a+1}}{2 \pi} e^{-2 a \gamma} \int r d r d \theta \frac{e^{i r \cos \theta}}{r^{2 a+2}}
$$


The integral can be readily computed,

$$
F(a)=e^{-2 \gamma a} \frac{\Gamma(1-a)}{\Gamma(1+a)}=1+O[a]^{3}
$$

It follows

$$
I(k)=-\pi \log \frac{k^{2}}{\mu^{2}}
$$

\section{Acknowledgments}

A.K. and A.O'B. would like to thank C.R. Graham for helpful conversations. The work of A.K. was supported in part by DOE contract \# DE-FG02-96-ER40956. The work of A.O'B. was supported by a Jack Kent Cooke Foundation scholarship. KS is supported by NWO.

\section{References}

[1] J. M. Maldacena, Adv. Theor. Math. Phys. 2, 231 (1998) [Int. J. Theor. Phys. 38, 1113 (1999)] [arXiv:hep-th/9711200].

[2] E. Witten, Adv. Theor. Math. Phys. 2, 253 (1998) [arXiv:hep-th/9802150].

[3] S. S. Gubser, I. R. Klebanov and A. M. Polyakov, Phys. Lett. B 428, 105 (1998) [arXiv:hep-th/9802109].

[4] M. Henningson and K. Skenderis, JHEP 9807, 023 (1998) [arXiv:hep-th/9806087].

[5] M. Henningson and K. Skenderis, Fortsch. Phys. 48, 125 (2000) [arXiv:hep-th/9812032].

[6] V. Balasubramanian and P. Kraus, Commun. Math. Phys. 208, 413 (1999) [arXiv:hep-th/9902121].

[7] S. de Haro, S. N. Solodukhin and K. Skenderis, Commun. Math. Phys. 217, 595 (2001) [arXiv:hep-th/0002230].

[8] C. R. Graham and E. Witten, Nucl. Phys. B 546, 52 (1999) [arXiv:hep-th/9901021].

[9] A. Karch and E. Katz, JHEP 0206, 043 (2002) [arXiv:hep-th/0205236].

[10] A. Karch and L. Randall, JHEP 0106, 063 (2001) [arXiv:hep-th/0105132].

[11] K. Skenderis and M. Taylor, JHEP 0206, 025 (2002) [arXiv:hep-th/0204054].

[12] N. R. Constable, J. Erdmenger, Z. Guralnik and I. Kirsch, Phys. Rev. D 68, 106007 (2003) [arXiv:hep-th/0211222].

[13] C. R. Graham, arXiv:math.dg/9909042.

[14] K. Skenderis, Class. Quant. Grav. 19, 5849 (2002) [arXiv:hep-th/0209067].

[15] C. Fefferman and C. Robin Graham, 'Conformal Invariants,' in Elie Cartan et les Mathematiques d'aujourd'hui (Asterique, 1985) 95.

[16] A. Petkou and K. Skenderis, Nucl. Phys. B 561, 100 (1999) [arXiv:hep-th/9906030]. 
[17] M. Bianchi, D. Z. Freedman and K. Skenderis, JHEP 0108, 041 (2001) [arXiv:hep-th/0105276]; Nucl. Phys. B 631, 159 (2002) [arXiv:hep-th/0112119].

[18] I. Papadimitriou and K. Skenderis, IRMA Lectures in Mathematical and Theoretical Physics 8, Eds O. Biquard, p. 73, arXiv:hep-th/0404176.

[19] I. Papadimitriou and K. Skenderis, JHEP 0410, 075 (2004) [arXiv:hep-th/0407071].

[20] M. Kruczenski, D. Mateos, R. C. Myers and D. J. Winters, JHEP 0307, 049 (2003) [arXiv:hep-th/0304032].

[21] N. J. Evans and J. P. Shock, Phys. Rev. D 70, 046002 (2004) [arXiv:hep-th/0403279].

[22] O. DeWolfe, D. Z. Freedman and H. Ooguri, Phys. Rev. D 66, 025009 (2002) [arXiv:hep-th/0111135].

[23] I. Papadimitriou and K. Skenderis, JHEP 0508, 004 (2005) [arXiv:hep-th/0505190].

[24] D. Arean and A. V. Ramallo, arXiv:hep-th/0602174.

[25] I. R. Klebanov and E. Witten, Nucl. Phys. B 556, 89 (1999) [arXiv:hep-th/9905104].

[26] D. Z. Freedman, S. D. Mathur, A. Matusis and L. Rastelli, Nucl. Phys. B 546, 96 (1999) [arXiv:hep-th/9804058].

[27] D. Z. Freedman, K. Johnson and J. I. Latorre, Nucl. Phys. B 371, 353 (1992).

[28] L. V. Avdeev, D. I. Kazakov and I. N. Kondrashuk, Int. J. Mod. Phys. A 9, 1067 (1994) [arXiv:hep-th/9302085]. 